\title{
DISCRIMINACIÓN Y RECONCILIACIÓN: COMUNIDAD JUDEO-ARGENTINA Y SU RELACIÓN CON EL RÉGIMEN MILITAR ARGENTINO (1976-1989)
}

\author{
Valeria Navarro \\ Licenciada en Historia (PUC) \\ Magíster en Estudios Internacionales (USACH) \\ soleilaviv@yahoo.es
}

\section{RESUMEN:}

Durante el complejo periodo del "Proceso de Reconstrucción Nacional" en Argentina (1976-1983) el ser judío y vivir dentro de una comunidad se convirtió en un desafío, que en muchos casos significaba un riesgo para la vida de quienes combinaban la militancia política y el judaísmo. En las siguientes páginas, se presentan las distintas formas de discriminación que vivieron los judíos durante el último de los regímenes militares argentino del siglo $\mathrm{XX}$, tanto desde el gobierno como dentro de la comunidad judía. Esto significó un fuerte impacto en la comunidad judía, en especial por la amplia dimensión de quienes fueron muertos durante el régimen militar por ser judíos. Finalmente, se explican algunos de los mecanismos de reparación de la memoria histórica utilizados dentro de la comunidad para tratar el tema de los detenidos desaparecidos de origen judío.

PALABRAS CLAVE:

Judíos - Argentina - Régimen Militar - Discriminación - Reconstrucción Memoria Histórica.

\section{ABSTRACT:}

During the complex time of the "National Reconstruction Process" in Argentina (1976-1983) to be a Jew and to live in the community context became a challenge, which in many cases meant a risk for those who combined both political militancy and Judaism. In the following pages we'll show the different types of discrimination that such Jews lived during the last Argentinean military regime of the $20^{\text {th }}$ century, both from the government and from inside the Jewish community. This had an enormous impact in the Jewish Community, especially due to the large dimension of those killed or tortured for being Jews. Finally, we'll explain some of the mechanism used to build the historic reconstruction of memory inside the Jewish community to deal the subject of Jewish disappeared prisoners.

\section{KEYWORDS:}

Jews - Argentina - Military Government - Discrimination - Historic Memory Reconstruction.

\section{Presentación}

Este ensayo proviene de la investigación realizada para obtener el Grado de Magíster en Estudios Internacionales (USACH), "Comunidades judías en Chile y Argentina durante los Regímenes Autoritarios. (1973-1991)", sobre el rol de las dirigencias comunitarias y su relación con el poder de facto. La tesis se enmarca en el proyecto Fondecyt 1050053 "Movimientos, Comunidades e Identidades Judías e Islámicas en América Latina: Los Casos de Argentina, Brasil y Chile desde 1991 hasta 2004", dirigido por el Prof. Isaac Caro. 
Valeria Navarro. Discriminación y reconciliación: comunidad Judeo-Argentina y su relación con el régimen militar argentino(1976-1989).

\section{La comunidad judía de Argentina}

Argentina es un caso particular en la composición judía de América Latina. Se destaca por concentrar la comunidad judía más numerosa, 300.000 para 1976, y un decrecimiento demográfico a la cifra de 184.000 para $2006^{1}$, según estimaciones del American Jewish Year Book; reúne también la mayor y más compleja red de instituciones y organizaciones judías. Como ente regulador y coordinador de las distintas instituciones, se forma la Delegación de Asociaciones Israelitas Argentinas (DAIA) en 1935 con el propósito de velar por los derechos humanos, el cumplimiento de las leyes de igualdad y libertad de culto, y en general para resguardar la vida y dignidad judía en Argentina ${ }^{2}$.

Así también, la comunidad judía argentina se convirtió a través del siglo XX en uno de los referentes del judaísmo latinoamericano, tanto para el continente como para las organizaciones internacionales, como la American Jewish Committee, que mantuvo hasta 1977 su representación para América Latina en Argentina.

\section{Política Argentina en 1976}

Para comprender la relación entre la comunidad judía y el régimen militar es necesario considerar que desde 1930 hubo cuatro golpes de estado y sólo dos presidentes lograron finalizar sus periodos de gobierno.

La figura del general Perón transformó el escenario político; su figura representa el nacionalismo, la industrialización y la justicia social ${ }^{3}$. Cuando llega al poder por tercera y última vez en 1973, su apoyo provenía tanto de los movimientos peronistas de izquierda, principalmente Montoneros, juventud radicalizada en lo social y lo cultural, de quién Perón se distancia, y los grupos paramilitares nacionalistas como la Triple A (Alianza Anticomunista Argentina), apoyada desde el ministerio de Bienestar Social.

La creciente violencia proveniente de ambos extremos del peronismo, así como una galopante crisis económica, y la ingobernabilidad del gobierno de María Estela (Isabel) Perón, permitió que las fuerzas armadas tomasen el poder con un alto apoyo social y de la opinión pública. Posteriormente se tornará un régimen gobernado por el terror, a causa de la fuerte represión ejercida.

\footnotetext{
${ }^{1}$ Ambas cifras provienen de la investigación demográfica que realiza el American Jewish Committe (Comité Judío Americano, una de las principales organizaciones judías en los Estados Unidos), sobre la población judía mundial desde 1899, publicados en los American Jewish Year Book, cuyos ejemplares se pueden encontrar en la siguiente página:

http://www.ajcarchives.org/main.php?Groupingld=40

Datos de 1976:

http://www.ajcarchives.org/AJC DATA/Files/1976 14 WJP.pdf

Datos de 2006:

http://www.ajcarchives.org/AJC DATA/Files/AJYB615.CV.pdf

${ }^{2}$ Herszkowich, 2006, pp. 46-47

${ }^{3}$ Cabe recordar que el General Juan Domingo Perón fue Presidente de Argentina en tres ocasiones: Su primer período abarcó el lapso 1946-1952. Reelecto en 1952 fue derrocado en 1955 por un golpe de Estado que instauró una dictadura militar (Revolución Libertadora). En este periodo estuvo exiliado en España, y regresó a la Argentina en 1973, año en que fue nuevamente electo por un tercer periodo para la presidencia del país. Esta última etapa finaliza con la muerte de Perón en julio de 1974
} 
Valeria Navarro. Discriminación y reconciliación: comunidad Judeo-Argentina y su relación con el régimen militar argentino(1976-1989).

Los tres tipos de discriminación durante la dictadura argentina

Antisemitismo proveniente de los grupos nazi-fascistas argentinos.

La llegada de la Junta Militar y la imposición del Proceso de Reorganización Nacional se tradujeron en tres tipos de discriminación para la Comunidad judía. En primer lugar, la discriminación y ofensa pública, provenientes de los grupos nacionalistas - fascistas, las editoriales Milicia y Odal, y la revista Cabildo cuyas publicaciones y artículos, considerando a los judíos como extranjeros, enemigos de la nación y promotores de todo lo que ellos identificaban como las lacras del mundo, tenían el siguiente tono: "Los dirigentes bolcheviques son casi todos judíos... las grandes estafas fueron perpetradas por judíos... ¿Por qué, si los judíos apenas llegan al 10\% de la población, son los autores de la mayoría de las estafas, quiebres fraudulentos, etc.?"4. Ante esto, la DAIA, se reúne con las autoridades militares para buscar alguna solución, planteando su preocupación por la distorsión que estos grupos hacían de la imagen del país en la opinión publica internacional, y también para presentarse no como "judíos temerosos, sino como argentinos preocupados". En las reuniones, especialmente en 1977, se solicitaba: "(1) una enérgica declaración en el más alto nivel gubernativo que ponga fin a este racismo desembozado que puede ser el preludio inevitable de futuras violencias, (2) dictar una legislación que reprima la promoción de la discriminación religiosa o racial en cualquier forma y por cualquier medio"". Esta legislación se consigue y en dos ocasiones se prohíben la circulación y venta de las revistas y publicaciones de las editoriales mencionadas.

Trato especial a los detenidos y desaparecidos judíos

Por parte del gobierno y del sistema de represión existió un ensañamiento particular con los prisioneros judíos. Esto ha sido estudiado en investigaciones específicas sobre el trato recibido por los prisioneros y desaparecidos judíos.

El primer indicio que explica la necesidad de realizar este tipo de estudios proviene de la alta cifra de detenidos desaparecidos judíos al final, registrado en la Comisión Nacional sobre la Desaparición de Personas (CONADEP), que registran un total inicial de 10.424 víctimas, de las que 1.296 son judíos, es decir un $12,43 \%$, lo que supera con creces el $0,5 \%$ calculado para el total de la población judía en correlación con la sociedad general ${ }^{7}$. También registra hechos de antisemitismo en los distintos centros de tortura.

El análisis del trato específico a los judíos muestra que se pueden identificar cinco tipos o momentos de la discriminación.

- El primer momento es la acción antisemita durante el secuestro o detención, en que, por ejemplo, se encuentran casos de baleos a libros en yiddish y hebreo, fuertes golpizas propinadas a quienes eran identificados como judíos y tratándolos peor, con duros insultos, en especial "judío".

- El segundo hito es la tortura y humillación específica para prisioneros judíos. Esto se registra tanto en el informe CONADEP, como en el informe de la Comisión Interamericana de Derechos Humanos

\footnotetext{
${ }^{4}$ Hitler o Lenin Editorial Milicia, citado en OJl, Boletín de Congreso Judío Latinoamericano, N914/76, segunda quincena de julio de 1976.

5 "La entrevista de Harguindeguy - DAIA", Mundo Israelita 24/7/1976

${ }^{6}$ OJl, Boletín del Congreso Judío Latinoamericano, N8/77, segunda quincena de mayo 1977.

7 Cifras de víctimas totales y víctimas judías en el listado de CONADEP, en Informe sobre la situación de los detenidosdesaparecidos judíos durante el genocidio perpetrado en Argentina 1976-1983, Informe elaborado por el Centro de Estudios Sociales de DAIA, noviembre 2007. Pág. 144
} 
Valeria Navarro

Discriminación y reconciliación: comunidad Judeo-Argentina y su relación con el régimen militar argentino(1976-1989).

realizado en 1980, en que se recopilan las crueles torturas aplicadas, por ejemplo, el rectoscopio, o las pintadas de svásticas en el cuerpo de los detenidos para golpearlos durante la ducha. No sólo la violencia física era más dura con los prisioneros judíos, sino también en el acoso verbal se les recordaba constantemente su religión, a través de ofensas como "judío bastardo", "judío de mierda", que los iban a hacer jabón como a sus familiares en Alemania ${ }^{8}$. Otro caso emblemático es el relato de una detenida que relata: "En una ocasión oímos a un perro ladrando en el pasillo y a alguien que le ordenaba ir de un lado a otro moviendo la cola. Pensamos que se trataba realmente de un perro. Pero no, era un ser humano, un hombre joven que tenía que fingir ser un perro porque había cometido el crimen de ser judío",

- El tercer caso es el uso de simbología, frases y lenguaje nazi durante la permanencia de los prisioneros en los campos de concentración, por ejemplo, la presencia de cuadros con la imagen de Hitler, svásticas en las ropas de los represores y dibujadas en los cuerpos de los prisioneros judíos y la repetición de grabaciones de los discursos de Hitler y líderes nazis durante todas las noches.

- Un cuarto elemento fueron los interrogatorios especiales a judíos, que buscaban información sobre las "campañas judías", como el Plan Andinia (invento de Walter Beveraggi Allende). Requerían información sobre los movimientos juveniles, las organizaciones comunitarias, el personal que trabajaba en los edificios de las instituciones judías, incluso la ideología y horarios de los funcionarios. Parecía un plan sistemático para realizar tareas de inteligencia sobre "las comunidades judías y sobre las personas de procedencia judía"10.

- El quinto elemento, era la expropiación de bienes de forma ilícita. Por ejemplo, se secuestraba a empresarios judíos para que "auto-liquidasen" sus bienes, para entregarlos a funcionarios ligados al régimen militar.

Una interpretación interesante sobre la particularidad de este trato hacia los judíos la presenta Jacobo Timerman, importante periodista que fue secuestrado, desaparecido, luego de ser detenido en su hogar, quién explica: "la tortura a un prisionero judío traía siempre un momento de divertimiento a las fuerzas de seguridad argentinas, un cierto momento de ocio", (...) "a un prisionero político se lo podía odiar porque estaba en el otro campo, pero también se podía intentar convencerlo, darlo vuelta, hacerle comprender que está equivocado, hacerle cambiar de bando, hacerlo trabajar para uno. ¿Pero cómo se puede cambiar a un judío? Es el odio eterno, interminable, perfecto, inevitable"11.

Discriminación comunitaria a familiares de detenidos y desaparecidos y la formación de instituciones paralelas sobre Derechos Humanos.

Un tercer tipo de discriminación existió dentro de los marcos comunitarios.

Esta se refiere a la indiferencia existente por parte de la dirigencia comunitaria hacia el problema que acongojaba a estas familias. Entrevistas realizadas por las comisiones que investigaban el trato especial a los detenidos judíos y el rol de la DAIA durante el régimen militar, denuncian, en primer lugar, que la DAIA nunca asumió como propio el tema de los desaparecidos, es decir, como un

\footnotetext{
${ }^{8}$ http://www.desaparecidos.org/arg/conadep/nuncamas/69.html

${ }^{9}$ Testimonio de Ana María Careaga, en "La dimensión judía en la represión durante el gobierno militar en la Argentina (19761983)", en El Antisemitismo en la Argentina. Centro Editor de América Latina. 2ª Edición corregida y aumentada, 1989, pág. 249 Testimonio de Jacobo Timerman, Testimonio de Pedro Miguel Vanrell, citado en Braylan, Feierstein, Galante, Jmelnizky, 2000, p. 307

${ }^{10}$ Informe sobre la situación de los detenidos-desaparecidos judíos durante el genocidio perpetrado en Argentina 1976-1983, Informe elaborado por el Centro de Estudios Sociales de DAIA, noviembre 2007.

${ }^{11}$ Timerman, 1982, p. 67
} 
Valeria Navarro. militar argentino(1976-1989).

problema que afectaba a la comunidad judía y a sus miembros, ni que las violaciones generalizadas a los derechos humanos fuera parte de lo que la dirigencia judía tuviese que hacerse cargo. "Pese a su naturaleza y la dramática dimensión, la cuestión parece no tener peso suficiente para ser ubicada y enfocada como un problema de la sociedad toda, y por ende, de la comunidad judía"12. En este mismo sentido, recuerdan que los funcionarios los recibían en pasillos, con tiempo insuficiente, de forma displicente e incluso, "deslizando opiniones peyorativas o descalificatorias sobre la "condición de judíos" de los desaparecidos"13, es decir, al comentario general de "por algo será", se agregaba la consideración de que, si hubiesen entregado una educación judía a los jóvenes, no se encontrarían en la situación que estaban ${ }^{14}$.

Quienes asumieron la defensa de los judíos detenidos y desaparecidos fueron el rabino conservador Marshall Meyer y el periodista Herman Schiller. El primero participó en la Asamblea Permanente por los Derechos Humanos $y$, tras el derrumbe del gobierno militar, asumió como miembro de la CONADEP. Sobre la comunidad organizada dice: "De ninguna manera puedo decir que la DAIA arriesgó algo por los derechos humanos. (...) llegó a formular alguna que otra declaración, pero hacer algo activo, no. Nunca los vi (...) en los años más duros de la dictadura militar, en alguna actividad concreta a favor de los detenidos-desaparecidos"15.

El periodista Herman Schiller fundó el periódico Nueva Presencia que deseaba convertirse en un nuevo referente comunitario y que integró el tema de los derechos humanos como parte primordial de su línea editorial. Desde 1979, Nueva Presencia comenzó a publicar petitorios e informes de las actividades de Madres de Plaza de Mayo ${ }^{16}$.

La constante perseverancia de Nueva Presencia en su denuncia de las violaciones a los derechos humanos y su incitación a la conciencia y participación de parte de las masas judías, es conocida y criticada por parte del gobierno militar. En una reunión del presidente de DAIA en 1983, Sión Cohen Imach, el presidente General Bignone le dice "Ah, con el asunto de los derechos humanos también ustedes nos dan con el hacha, nos dan fuerte, a través de vuestro periódico Nueva Presencia" 17.

El vacío existente en el espacio comunitario para reconfortar, solidarizar y ayudar a los familiares de detenidos y desaparecidos judíos se comenzó a suplir con la formación del Movimiento Judío por los Derechos Humanos. Fundado por Herman Schiller, Marshall Meyer y varios padres de detenidos, comenzó a funcionar en 1982. Este "se pronunciaba contra la connivencia entre instituciones judías y situación represiva, y su silencio ante las desapariciones de personas" ${ }^{18}$. Su propósito era luchar por el respeto de los derechos humanos y el esclarecimiento de la situación de los detenidos y desaparecidos. Desde una cosmovisión judía del mundo, explican: "queremos luchar contra todas las lacras de la sociedad en que residimos- la tortura, los secuestros, el hambre, las desapariciones -

\footnotetext{
12 “Replica al 'Informe especial sobre detenidos-desaparecidos judíos, 1976-1983', publicado por la DAIA”, Nueva Presencia, $2 / 11 / 1984$

13 "Replica al 'Informe especial sobre detenidos-desaparecidos judíos, 1976-1983', publicado por la DAIA”, Nueva Presencia, $2 / 11 / 1984$

${ }^{14}$ Entrevista a Marcos Weinstein, realizada por Daniel Feierstein, 11/6/1999

${ }^{15}$ Nueva Presencia, "Marshall T. Meyer: Estamos investigando a fondo y todos los culpables de las atrocidades cometidas por la dictadura militar serán castigados", 17/2/1984

16 "Por los desaparecidos", Nueva Presencia, 19/9/1980

17 “Bignone se quejó ante la DAIA por la línea periodística de ‘Nueva Presencia'. 2/9/1983

${ }^{18}$ Marcos Weinstein, "Los Padres de Plaza de Mayo: Traslado, Eufemismo de Muerte". http://www.madresfundadoras.org.ar/memoria.shtml?sh itm=269f421afd2e9e963e74752e3e0e51b3
} 
desde nuestra propia identidad y por ello resulta muy importante la simpatía que el MJDH ha generado en todas las organizaciones de derechos humanos, comenzando con las Madres de Plaza de Mayo"19.

\section{Iniciativas de memoria y reconciliación.}

Por reconciliación se consideran las iniciativas tendientes a reconstruir el discurso comunitario, integrando la situación particular de los detenidos y desaparecidos judíos durante el régimen militar, como parte de su propia narración e identidad judeo-argentina. Se considera como parte de la reconstrucción de la memoria y evitar "la destrucción deliberada del registro histórico", en que sólo las pruebas, las evidencias cuidan la veracidad de los hechos acaecidos ${ }^{20}$.

El primer hecho concreto por parte de DAIA corresponde al polémico Informe Especial sobre detenidos y desaparecidos judíos entre 1976 y 1983, realizado para complementar el informe de la CONADEP en 1984.

Al mismo tiempo que DAIA realiza su informe, se entrega un reporte El trato recibido por detenidos $y$ desaparecidos durante la dictadura militar argentina, 1973-1986. Los prisioneros judíos, por parte del Comité Israelí de Familiares de Detenidos Desaparecidos en Argentina a la CONADEP, que recopila testimonios y entrevistas sobre el trato especial recibido por los judíos en la dictadura.

Paralelamente, se realizan los estudios de Edward Kaufmann y Beatriz Cymberknopf, La dimensión judía en la represión durante el gobierno militar en la Argentina, y de Ignacio Klich, Política comunitaria durante las Juntas Militares argentinas: la DAIA durante el Proceso de Reorganización Nacional. Los artículos fueron publicados en el libro Antisemitismo en la Argentina, que reúne diversos testimonios, documentos y artículos sobre la situación de los detenidos y de la Comunidad Judía durante el régimen militar.

En Israel, el Comité Israelí de Familiares de Detenidos Desaparecidos en Argentina presiona para que se forme una comisión del gobierno israelí, para identificar cuál fue el rol y actitud de Israel ante la situación de los judíos en Argentina durante la dictadura. Esta se concreta en el año $2000^{21}$.

También en Israel, se forma en 1998 la Asociación de Familiares de Desaparecidos Judíos en Argentina, para lograr la participación de Israel en la recuperación de los cuerpos de los desaparecidos y poder enterrarlos bajo el ritual judío, y conseguir apoyo en la búsqueda de los 20 niños judíos nacidos en cautiverio ${ }^{22}$. Esta organización colaboró con AMIA, en la publicación La violación de los derechos humanos de argentinos judíos bajo el régimen militar, de la Comisión Solidaria con familiares de presos y desaparecidos en la Argentina $^{23}$.

\footnotetext{
19 "Voces de aliento, criticas y sugerencias", Respuesta a la DAIA I, de Simon Lewintal. Nueva Presencia, 28/10/1983

${ }^{20}$ Haym Yerushalmi, 2002, p. 138

21 "Desaparecidos judíos: viajamos para garantizar que se cumplan las recomendaciones de la comisión”, Septiembre 2003, "Desaparecidos judíos en Argentina, Informe y conclusiones de la comisión israelí", diciembre 2002. Nueva Sión. http://www.clarin.com/diario/2001/01/05/p-01501.htm

22 "Asociación de familiares de desaparecidos judíos en la argentina”. Marcos Weinstein. Nueva Sión, 10/10/2001

${ }^{23}$ Citado en: Co. So. Fam, Comisión de Solidaridad con Familiares de Presos y Desaparecidos en la Argentina (Barcelona), La violación de los derechos humanos de argentinos judíos bajo el régimen militar (1976-1983), Editorial Milá, Buenos Aires, Argentina, 2006.

http://orientemedio.net/default.asp?CatelD $=13 \&$ cat=13\&dismode=article\&apage=2\&artid=3018
} 
Valeria Navarro. Discriminación y reconciliación: comunidad Judeo-Argentina y su relación con el régimen militar argentino(1976-1989).

Por parte de la DAIA, el centro de Estudios Sociales realizó dos investigaciones, una en 1999 para ser entregada al juez Baltasar Garzón, sobre la causa de genocidio contra la Junta Militar ${ }^{24}$ y la segunda, en 2003, para investigar el rol de DAIA durante la Dictadura, y que se concretó en la publicación del libro Informe sobre la situación de los detenidos-desaparecidos judíos durante el genocidio perpetrado en Argentina 1976-1983, y también en la construcción del Archivo DAIA-CES, sobre el rol de la DAIA en el periodo.

La reivindicación de los detenidos como parte de la memoria de la comunidad judía argentina, y en especial por parte de la dirigencia, se evidencia con dos actos simbólicos. El primero es la instauración de un bajo relieve de la artista y madre de detenido desaparecido Sara Brodsky, en el nuevo edificio de la AMIA. El acto se realiza en el 2004 con la presencia de representantes del movimiento judío por los derechos humanos, madres y abuelas de Plaza de Mayo, autoridades comunitarias, entre otros ${ }^{25}$. Para los miembros del MJDH esto simbolizaba que se están "revirtiendo actitudes de las instituciones oficiales de la comunidad judeoargentina respecto de sus desaparecidos -quienes fueron colocados en la categoría de 'ajenos al cuerpo social' (...) significa la recuperación de la identidad y la presencia, y la posibilidad de iniciar un cierre del duelo en suspenso"26.

El segundo corresponde al premio DAIA 2005 entregado a las organizaciones Poder ciudadano, Asamblea Permanente por los Derechos Humanos, B'nei B'rith Argentina y Movimiento Judío por los Derechos Humanos, por su labor "en el campo de los derechos humanos y el fortalecimiento de la democracia",27.

A pesar del interés de reivindicarse por parte de la DAIA, el MJDH no aceptó el premio, realizando un duro y crítico discurso durante la ceremonia de entrega del premio, en que se recordaba y recalcaba cómo fueron acusados de favorecer el antisemitismo por realizar actos públicos, algunos dichos polémicos de dirigentes, que consideraban como terroristas a los desaparecidos y su connivencia con el poder militar de facto ${ }^{28}$.

A través de estas distintas acciones, el discurso de la comunidad judía en general y de la dirigencia comunitaria en particular, se fue modificando durante el periodo de la renovada democracia argentina, incluyendo en su memoria, la situación de los detenidos y desaparecidos judíos, así como de las violaciones de los derechos humanos durante el régimen militar entre 1976 y 1983.

\footnotetext{
${ }^{24} \mathrm{http}: / /$ www.daia.org. ar/vernota. asp?id area=4\&id sec=9\&id item=303\&pagina=1 http://www.mfa.gov.il/desaparecidos/pdfspen/InformeDesapDAlAcast.pdf

${ }^{25}$ “'La AMIA reivindicó a los desaparecidos judíos”, revista Emet, La verdad de la Gente, marzo, 2004 http://ar.geocities.com/programaemet/Emetdigitales/2004/42-marzo2004ii.doc

${ }^{26}$ Dr. Marcos Wainstein, presidente de la Asociación de Familiares de Desaparecidos Judíos en la Argentina, Ídem.

${ }^{27}$ http://www.avina.net/web/siteavina.nsf/NoticiasCat/5FE869D3098F227B0325731D00523332?OpenDocument\&idioma=spa\&si stema $=1$ http://www.pagina12.com.ar/diario/elpais/1-55956-2005-09-02.html

${ }^{28} \mathrm{http}: / /$ www.lavozylaopinion.com.ar/cgi-bin/medios/vernota.cgi?medio=lavoz\&numero=Octubre2005\&nota =Octubre2005-18
} 


\section{REFERENCIAS BIBLIOGRÁFICAS.}

AVNI Haim, "Antisemitismo en Argentina: Las dimensiones del peligro", EN SENKMAN Leonardo y SZNAJDER Mario (eds), El legado del autoritarismo: derechos humanos y antisemitismo en la Argentina contemporánea. Editorial Instituto Harry S. Truman, Universidad Hebrea de Jerusalem / Grupo Editorial Latinoamericano, Buenos Aires, Argentina, 1995.

"La emancipación y la educación judía a la sombra de los regímenes militares y populistas (19661984)", INDICE. Revista de Ciencias Sociales, Año XXXVII, N24, Delegación de Asociaciones Israelitas Argentinas (DAIA)-Centro de Estudios Sociales, Buenos Aires, 2007.

BETHELL Leslie editor, "Argentina, 1930-1946”, Historia de América Latina, tomo 15. El cono sur desde 1930. Editorial Crítica, Barcelona, 2002.

BRAYLAN Marisa, FEIERSTEIN Daniel, GALANTE Miguel, JMELNIZKY Adrián, "Informe sobre situación de los detenidos-desaparecidos judíos durante el genocidio perpetrado en Argentina", INDICE. Revista de Ciencias Sociales, año XXXIV N20, Delegación de Asociaciones Israel itas Argentinas (DAIA)-Centro de Estudios Sociales, Buenos Aires. 2000.

BRENMAN Dario y EFRON Gustavo, Reseña de GUTMAN Daniel: Tacuara, historia de la primera guerrilla urbana argentina., Editorial Vergara, Buenos Aires, 2003, pp. 333. EN: Informe sobre antisemitismo 2003, Delegación de Asociaciones Israelitas Argentinas (DAIA)-Centro de Estudios Sociales, Buenos Aires., 2004.

CO. SO. FAM, Comisión de Solidaridad con Familiares de Presos y Desaparecidos en la Argentina (Barcelona), La violación de los derechos humanos de argentinos judíos bajo el régimen militar (1976-1983), Editorial Milá, Buenos Aires, Argentina, 2006.

FEIERSTEIN Ricardo, "¿Critica teórica o función concreta? Los Intelectuales y la Comunidad", EN Contraexilio y Mestizaje. Ser judío en la Argentina. Editorial Mila. 1996. Buenos Aires, Argentina.

, Historia de los Judíos Argentinos, Ed. Ameghinho, Buenos Aires, Argentina, 1999.

HERSZKOWICH Enrique, Historia de la Comunidad Judía Argentina. Delegación de Asociaciones Israelitas Argentinas (DAIA)-Centro de Estudios Sociales, Buenos Aires., 2006.

Informe Especial. Sobre detenidos y desaparecidos judíos. 1976-1983, Delegación de Asociaciones Israelitas Argentinas (DAIA)-Centro de Estudios Sociales, Buenos Aires.., enero 1984.

Informe sobre la situación de los detenidos-desaparecidos judíos durante el genocidio perpetrado en Argentina 1976-1983, Informe elaborado por Delegación de Asociaciones Israelitas Argentinas (DAIA)Centro de Estudios Sociales, Buenos Aires., noviembre 2007.

JMELNIZKY Adrián, "EI Peronismo y la inmigración a la Argentina luego de la Segunda Guerra Mundial", Nuestra Memoria, Museo del Holocausto Shoá, Fundación Memoria del Holocausto, Buenos Aires, Argentina, Año XI, № 25, Junio 2005.

KAUFMAN Edy y CYMBERKNOPF Beatriz, "La dimensión judía en la represión durante el gobierno militar en la Argentina (1976-1983), EN El Antisemitismo en la Argentina. Centro Editor de América Latina. $2^{a}$ Edición corregida y aumentada, 1989. 
Valeria Navarro. Discriminación y reconciliación: comunidad Judeo-Argentina y su relación con el régimen militar argentino(1976-1989).

KLICH Ignacio, "Política Comunitaria durante las Juntas Militares argentinas: La DAIA durante el Proceso de Reorganización Nacional", EN El Antisemitismo en la Argentina. Centro Editor de América Latina. $2^{\mathrm{a}}$ Edición corregida y aumentada, 1989.

LAIKIN ELKIN Judith, Jews of the Latin American Republics. The University of the North Carolina Press. USA, 1980.

LVOVICH Daniel, BOHOSLAVSKY Ernesto, "Los judíos y la política en Iberoamérica en el siglo XX", EN REYES MATE y FORSTER Ricardo (eds.) El judaísmo en Iberoamérica. Edición de, Editorial Trotta, Madrid, 2007.

"Perón, la comunidad judía argentina y la cuestión del antisemitismo", INDICE. Revista de Ciencias Sociales, año XXXVII N24, Delegación de Asociaciones Israe litas Argentinas (DAIA)-Centro de Estudios Sociales, Buenos Aires 2007.

MIRELMAN Víctor A., "Las organizaciones internacionales judías ante la represión y el antisemitismo en Argentina", EN SENKMAN Leonardo y SZNAJDER Mario (eds), El legado del autoritarismo: derechos humanos y antisemitismo en la Argentina contemporánea. Editorial Instituto Harry S. Truman, Universidad Hebrea de Jerusalem / Grupo Editorial Latinoamericano, Buenos Aires, Argentina, 1995.

NOVARO Marcos y PALERMO Vicente, Historia Argentina. La Dictadura Militar 1976-1983. Editorial Paidos. Buenos Aires 2003.

QUIROGA Hugo, El Tiempo del Proceso. Conflictos y coincidencias entre políticos y militares 1976-1983. Editorial Fundación Ross-HomoSapiens, Segunda Edición, 2004.

REIN Raanan, "El fracaso de la peronización de la colectividad judía”, Nuestra Memoria, Museo del Holocausto Shoá, Fundación Memoria del Holocausto, Buenos Aires, Argentina, Año XI, №25, Junio 2005.

RIZ Liliana de, Historia Argentina. La Política en suspenso 1966/1976. Editorial Paidos, Buenos Aires, Argentina, 2000.

SENKMAN Leonardo, "El antisemitismo bajo dos experiencias democráticas: Argentina 1959/1966 y 1973/1976", EN El Antisemitismo en la Argentina. Centro Editor de América Latina. 2a Edición corregida y aumentada, 1989

TIMERMAN Jacobo, Preso sin nombre, celda sin número. El Cid editor, Buenos Aires, 1982.

WAISMAN Carlos H., "La ideología del nacionalismo de derecha en Argentina: El capitalismo, el socialismo y los judíos, EN El Antisemitismo en la Argentina. Centro Editor de América Latina. 2 $2^{\mathrm{a}}$ Edición corregida y aumentada, 1989

HAYM YERUSHALMI Yosef, Zajor, La Historia judía y la memoria judía. Editorial Anthropos, España, 2002 (traducción de la $3^{\text {a }}$ edición de 1996).

Archivos, documentos, entrevistas

Archivo Histórico: "El rol de la DAIA frente a la dictadura militar argentina (1976-1983)"

Entrevistas:

Argentina:

Herman Schiller, Beatriz Gurevich, Adrián Krupnik, Marcos Doño, Daniel Lvovich, Ricardo Feierstein.

\section{Diarios y Revistas}

CUADERNOS JUDAICOS-ISSN: 0718-8749

№26, diciembre 2009 
Valeria Navarro.

Discriminación y reconciliación: comunidad Judeo-Argentina y su relación con el régimen militar argentino(1976-1989).

\section{En papel}

Argentina:

Mundo Israelita; OJI, Boletín Congreso Judío Latinoamericano; Nueva Sión; Nueva Presencia; Convicción; La Razón.

\section{En Internet}

American Jewish Year Book 1939-1940; 1960; 1974-1975; 1978; 1986; 2002. http://www.ajcarchives.org/ Emet, La verdad de la Gente http://ar.geocities.com/programaemet/Emetdigitales/2004/42-marzo2004ii.doc

La Voz y la Opinión. www.lavozylaopinion.com.ar, http://www.desaparecidos.org/ , http://www.avina.net 